\title{
Geometric Calibration for Multi-projector Tiled Display Based on Vanishing Point Theory
}

\author{
Yuan Guodong, Qin Kaihuai, and Hu Wei \\ Department of Computer Science and Technology, Tsinghua University, Beijing, China \\ \{ygd02@mails., qkh-dcs@, huw02@mails.\}tsinghua.edu.cn
}

\begin{abstract}
In this paper, we present a new geometric calibration method for multi-projector tiled display. Firstly, one circular feature template is attached onto a planar screen and a single un-calibrated camera observes the circular feature template and the tiled arrangement for each projector alternatively. Secondly, geometric coordinates on the planar screen for all features in each tile are computed according to the two vanishing points from the perspective template image. Thirdly, the inscribed quadrangle region in the planar screen space is calculated as the effectively region of the tiled display. Finally the geometric alignment is achieved by the correspondence between the quadrangular mesh in the projector space and the one in the display space. Our algorithm removes the limitation against the parallelism between camera image plane and planar screen plane and improves the convenience to deploy a multi-projector display system. The provided experimental results demonstrate the validity and efficiency of our new method.
\end{abstract}

\section{Introduction}

In recent years, many commercial multi-projector systems have been widely available, such as ImmersiveWall, PowerWall, DataWall and etc. While the high cost and technical expertise have restricted their usage to only a few large institutions and well-funded universities. However, many emerging classes of users do not have enough budgets and expertise. So it is imperative to offer new geometric calibration algorithm, which can be easily to deploy a low-cost tiled display system in existing spaces.

In this paper, a new geometric calibration algorithm for multi-projector display system is proposed. It uses an un-calibrated camera to observe a template and a set of overlapping projectors alternatively. From the template image, the three vanishing points in a 3D space are calculated. Then the coordinate of each feature on the planar screen can be reconstructed according to the vanishing point theory. From such information, the necessary image-based corrections to construct a seamless image mosaic across the projector array can be computed. By contrast with [1], the camera's orientation and its position can be laid more freely, which improves the flexibility and the configurability of multi-projector display system. Various experimental results have demonstrated the efficiency and the accurateness of the geometric calibration algorithm. 


\section{Related Work}

Geometric calibration is a fundamental step to achieve the seamless and smooth visual effect for multi-projector tiled display. As for planar screen, automatic software method for geometric calibration has been independently studied using calibrated cameras [2] [3] besides manually adjusting mean. Likai's group utilizes the camera homography to address the limitation of camera's field-of-view and to improve the scalability of multi-projector tiled system [4].To significantly reduce the infrastructure cost, R. Raskar provided a geometric calibration that could adapt to a given projector array configuration [5]. This method must compute two kinds of homographies: camera-projector homography and projector-screen homography. To simplify the computation of geometric calibration further, $\mathrm{M}$. Brown et al [1] assumed that a static camera was placed in a position to observe all the output of multiple projectors, where the camera image plane is parallel to the planar screen. So it is unnecessary to calculate the above two kinds of homography. But this constraint reduces the visual range, which a camera can observe.

\section{The Algorithm}

First, a physical small pattern is attached to the planar screen to remove the constraint of the parallelism between the camera image plane and the planar screen,. Then a single un-calibrated camera is placed in the position where the entire output of all projectors can be captured. Finally, equally-spaced circles are projected from each of the projectors alternatively, so the camera can capture these images both from each projector and the small pattern at the same position. Figure 1 (a) $\sim$ (e) show the template and these observed projectors.



(a)

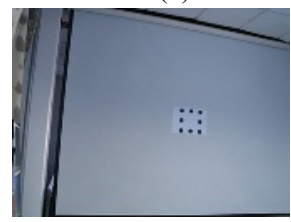

(e)

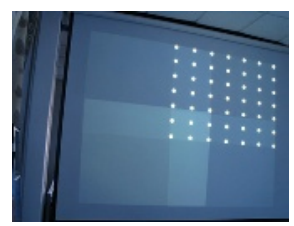

(b)

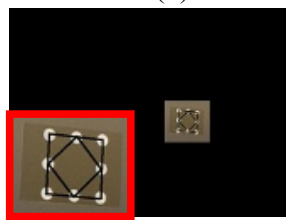

(f)

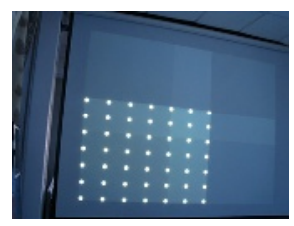

(c)

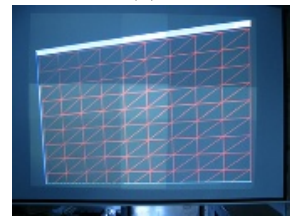

(g)

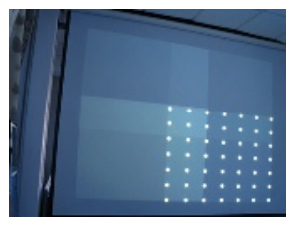

(d)



(h)

Fig. 1. (a,b,c,d) The captured images of projectors. (e) The pattern image. (f) The processed pattern image. (g) The geometric alignment result using the observed images directly. (h) The geometric calibration results by our method. 


\subsection{Calculating the Feature's Coordinate in the Planar Screen}

From the perspective template image shown in Figure 1 (f), the two vanishing points are calculated. Then the third vanishing point is calculated according to the perspective vanishing point theory. From that, a 3D coordinate system O_XYZ is created and the plane of the projection screen is denoted as the XOZ plane. For each observed projector, the centroid of each equally-spaced feature circle is calculated to get the 3D position vector of each feature in the display space on the basis of perspective projection. Because these $3 \mathrm{D}$ vectors are in the same plane, they can be denoted as $2 \mathrm{D}$ vector.

\subsection{Computing the Inscribed Display Region}

Physical display region in the planar screen is the union of the output of all projectors. As mentioned in [5], finding the maximum optimal inscribed rectangle for a union of quadrilaterals is a $2 \mathrm{D}$ constrained linear optimization problem. Due that the problem is NP hard, the effective display region $\mathrm{R}$ can be calculated by using a simple caseby-case heuristic. In the planar display space, the features of each projector $i$ can be formed to a quadrilateral mesh $M_{i}$. Then the intersection region between $R$ and $M_{i}$ can be re-meshed into a new $m \times n$ quadrilateral mesh $I M_{i}$.

\subsection{Calibration Computation Between Projector Space and Display Space}

For each projector $i$, the equally-spaced features is also formed to a quadrilateral mesh $P M_{i}$ in projector space according to the same rule as in the display space. To achieve the geometric calibration for the multiple projectors, the texture and geometric coordinate for each vertex of the mesh $I M_{i}$ in the projector space are needed. The details of calibration calculation are discussed as follows.

First, the minimum bounding rectangle $R_{i}$ for the mesh $I M_{i}$ in the display space is calculated. Second, the texture size for this projector $i$ and the texture coordinate for each vertex by a simple $2 \mathrm{D}$ window transformation are calculated. Third, the one-toone correspondence between the mesh $M_{i}$ in projector space and the mesh $P M_{i}$ in the display space is constructed to compute the geometric coordinate for each vertex.. Finally, the texture coordinate can be computed according to the theory of quadrilateral area coordinates. Once the size of the texture to be shown, the geometric and texture coordinate have been calculated for each projector, the seamless tiled display is achieved by rendering the quadrilateral mesh $P I M_{i}$ binding the texture using opengl.

\section{Experimental Results and Conclusion}

The multi-projector tiled display system used in the experiments was composed of four Sony CX80 projectors, a digital camera on a single PC and our new geometric calibration algorithm. As shown in Figure 1(g), geometric alignment can be achieved by using the observed images directly. But geometric distortion occurs in the display region because of the projective distortion between the captured geometry and physical output geometry of the projectors. Figure 1(h) shows that the geometric calibration results using our algorithm. 
To a planar projection screen, a new geometric calibration algorithm for multiprojector tiled display on the basis of the theories of vanishing point and quadrilateral area coordinate is presented. There are two main advantages of this algorithm. One is that it can easily achieve sub-pixel alignment accuracy. The other is that it breaks through the limitation of the parallelism between the camera image plane and the planar screen plane, thus the camera can capture much greater view-of-field in the same condition. From the experimental results, the new algorithm improves the flexibility and scalability of multi-projector tiled display system.

\section{Acknowledgements}

This work received supports from the National Natural Science Foundation of China (Grant No. 60473112), the Specialized Research Fund for the Doctoral Program of Higher Education of China (20030003053).

\section{References}

1. Michael S. Brown, W. Brent Seales: Incorporating Geometric Registration with PC-Cluster Rendering For Flexible Tiled Displays. Int. J. Image Graphics 4(4): 683-700 (2004)

2. R.Surati, Scalable Self-Calibrating Display Technology for Seamless Large-Scale Displays, $\mathrm{PhD}$ thesis, Dept. of Electrical Engineering and Computer Science, Massachusetts Institute of Technology, Cambridge, Mass., 1999.

3. R. Raskar et al., "Multi-Projector Displays Using Camera-Based Registration," Proc. of IEEE Visualization 1999, ACM Press, New York, Oct. 1999.

4. H. Chen, R. Sukthankar, and G. Wallace, "Scalable Alignment of Large-Format MultiProjector Displays Using Camera Homography Trees," Proc. IEEE Visualization 2002, pp. 339-346, 2002.

5. R. Raskar, J. van Barr, and J.X. Chai. A low-cost projector mosaic with fast registration. In ACCV 2002. 